\title{
Gestión logística en los departamentos de control de estudios de las instituciones públicas de educación superior
}

\author{
Logistics management in the departments of control of studies of the public institutions \\ of higher education
}

Gestão da logística nos departamentos de controle de estudos das instituições públicas de ensino superior

Recibido: octubre 2019

Arbitrado: noviembre 2019

Aceptado: diciembre 2019

Publicado: enero 2021

\author{
Elaine Alejandra Bermúdez Maldonado \\ elaber02@hotmail.com \\ https://orcid.org/0000-0003-3718-3739
}

Universidad politécnica territorial del estado Zulia, Venezuela
RESUMEN

La investigación tuvo como objetivo describir la gestión logística en los departamentos de control de estudios de las instituciones públicas de educación superior en la Costa Oriental del Lago. Metodológicamente se caracterizó como una investigación de tipo descriptiva, con un diseño no experimental, de campo y transeccional. La población estuvo conformada por 12 sujetos, los cuales representan el personal que labora en los departamentos de control de estudios de la Universidad del Zulia (LUZ), el Instituto Universitario de Tecnología de Cabimas (IUTC) y la Universidad Experimental Rafael María Baralt (UNERMB), respectivamente. La técnica que se utilizó fue la encuesta. Para la recolección de los datos se diseñó un instrumento cuestionario tipo Likert compuesto por 56 ítems, dicho instrumento fue validado previamente por cinco 5 expertos en el área. Los resultados obtenidos permitieron concluir que la variable gestión logística de las instituciones estudiadas se desarrolla con un moderado nivel de cumplimiento.

Palabras clave: Gestión logística; departamento; control de estudio; instituciones, educación
The objective of the research was to describe the logistics management in the departments of control of studies of the public institutions of higher education in the Eastern Coast of the Lake. Methodologically, it was characterized as a descriptive research, with a non-experimental, field and transectional design. The population consisted of 12 subjects, which represent the personnel working in the study control departments of the University of Zulia (LUZ), the University Institute of Technology of Cabimas (IUTC) and the Rafael María Baralt Experimental University (UNERMB), respectively. The technique used was the survey. For data collection, a Likert-type questionnaire instrument composed of 56 items was designed, this instrument was previously validated by five experts in the area. The results obtained allowed to conclude that the variable logistics management of the institutions studied is developed with a moderate level of compliance.

Key words: Logistics management; Department; study control; institutions, education
RESUMO

O objetivo da pesquisa foi descrever a gestão logística nos departamentos de controle de estudos das instituições públicas de ensino superior da Costa Leste do Lago. Metodologicamente, caracterizou-se como uma pesquisa descritiva, com desenho não experimental, de campo e transversal. A população foi composta por 12 sujeitos, que representam o pessoal que trabalha nos departamentos de controle de estudos da Universidade de Zulia (LUZ), do Instituto Universitário de Tecnologia de Cabimas (IUTC) e da Universidade Experimental Rafael María Baralt (UNERMB), respectivamente. A técnica utilizada foi o survey. Para a coleta de dados, foi elaborado um instrumento de questionário do tipo Likert composto por 56 itens, este instrumento foi previamente validado por cinco especialistas na área. Os resultados obtidos permitiram concluir que a variável gestão logística das instituições estudadas é desenvolvida com um nível de conformidade moderado.

Palavras chave: Logistics management; Departamento; controle do estudo; instituições, educação 


\section{INTRODUCCIÓN}

EDhas la actualidad existen retos importantes que exige a la sociedad actual la necesidad de promover la gestión logística dentro de las organizaciones, esto va desde los dueños hasta aquellos directivos o gerentes que se encargan de gestionar las actividades logísticas. En tal sentido, se requiere contar con personal capacitado, apto para asumir riesgos y gestionar con éxito los procesos de cambio que garanticen el crecimiento continuo de la rentabilidad y utilidad de la empresa, así como también satisfacer los requerimientos de los clientes brindando un servicio de calidad a la altura de sus necesidades.

Sin duda, la gestión logística es una parte fundamental en cualquier actividad económica, encargada de gestionar la planificación, organización, ejecución y control de los procesos y de esta manera alcanzar los objetivos planteados en las organizaciones. Es mejorar continuamente los procesos inmersos en las aéreas de producción, almacenamiento, distribución; entre otras. Además, permite trabajar de manera conjunta, organizada, creativa, eficiente, con valores, ética y con una moral comprometida con el desarrollo humano.

En efecto, las organizaciones hoy en día están obligadas a definir estrategias que permitan el acceso al mundo competitivo de los mercados y si éstas no van acompañadas de las herramientas de gestión que garanticen su materialización, los esfuerzos para obtener éxito en la administración de cualquier empresa serán inútiles.

Al respecto Rementeria (2008, p. 1), define la gestión como la "actividad profesional tendiente a establecer los objetivos y medios de su realización, a precisar la organización de sistemas, a elaborar la estrategia del desarrollo y a ejecutar la gestión del personal". Es decir, que por gestión se entiende la dirección de las acciones que contribuyan a tomar decisiones orientadas a alcanzar los objetivos trazados, medir los resultados obtenidos, para finalmente orientar la acción hacia la mejora permanente del sistema.

De esta manera, puede inferirse que la gestión es el proceso que coadyuva a la organización en el logro de sus metas, para lo cual es preciso que los recursos estén disponibles en el tiempo justo. De igual manera, la logística debe coordinar todos los procesos, desde el primer proveedor hasta que el producto llega al cliente.

Según Council of Supply Chain of Management Professionals (CSCMP) (2013), la logística es aquella parte de la gestión de la cadena de suministro que planifica, implementa y controla el flujo y el almacenamiento eficaz y eficiente de los bienes, servicios e información relacionada desde el punto de origen al de consumo con el propósito de satisfacer los requerimientos de los consumidores.

Según Ballou (2004), esta definición es excelente, porque transmite la idea de que los flujos del producto deben ser manejados desde el punto donde se encuentran como materias primas hasta el punto donde finalmente son descartados. Para el autor, la logística también se ocupa del flujo de los servicios, así como de los bienes físicos, un área de crecientes oportunidades de mejora. Es decir, es la encargada de la distribución eficiente de los productos de una determinada empresa con un menor costo y un excelente servicio al cliente, desde que llega la materia prima hasta que se distribuye el producto o se presta un servicio. 
A partir de lo planteado por los autores Rementeria (2008), CSCMP (2013) y Ballou (2004), se puede inferir que la gestión logística es la planeación, ejecución, control y estrategia utilizada para alcanzar los objetivos planteados en las organizaciones. Es decir, el mejoramiento continuo de los procesos en las aéreas de producción, almacenamiento y distribución, entre otras, para que el producto terminado sea entregado al cliente en las mejores condiciones, en un determinado tiempo y a un precio razonable.

De igual manera, desde la perspectiva gerencial la gestión logística tiene como objetivo proveer los materiales necesarios para el rendimiento óptimo de las operaciones en una organización, al tomar en consideración los factores de demanda, costos y tiempo. El alcance de la gestión logística debe enfocar todo lo referente a los procesos gerenciales; planificar, organizar, ejecutar y controlar todas las actividades que sean necesarias para conseguir los niveles deseados de servicio y calidad al menor costo posible. El rol del gerente debe estar orientado a facilitar las herramientas necesarias para el mejoramiento continuo de estos procesos.

En los países de América Latina, como Venezuela, tal como lo señalan Casanova y Cuatrecasas (2010), se realizan esfuerzos para adecuar las industrias u organizaciones a los requerimientos internacionales, a través de nuevos modelos de gestión. Con el devenir del tiempo los modelos de gestión propios del sector productivo han migrado hacia la gestión de organizaciones sin fines de lucro como lo es, por principios, la educación (Drucker, 1990; Glatter, 1999; citado por Casassus, 2002).

En efecto, para las instituciones educativas, en especial las públicas, que buscan optimizar los procesos administrativos que se generan dentro de sus instalaciones, la gestión logística pasa a ser protagonista de primer orden, ya que a través de esta logran planificar, organizar, ejecutar y controlar el funcionamiento de sus actividades; brindar un mejor servicio y gestionar todo el caudal de la información que parte desde el establecimientos de objetivos hasta lograr satisfacer en sus requerimientos a la población estudiantil y docentes que en ellas hacen vida.

Así, la gestión logística se constituye en una herramienta indispensable para las instituciones públicas de educación superior en la Costa Oriental del Lago, que son casas de estudio que cuentan con un gran número de estudiantes de la región y foráneos. Dentro de estas instituciones se desarrollan diversos procesos de altos niveles de exigencia que requieren ser controlados, tales como: inscripciones, apoyo docente, revisión de notas, expedientes, nóminas; entre otros, los cuales necesitan una planificación y, procura de materiales y equipos para su desarrollo eficiente.

Estas instituciones cuentan con departamentos de control de estudios que tienen la finalidad de iniciar y cerrar los expedientes académicos pertenecientes a los estudiantes, así como de los egresados; además, velar por el cumplimiento de los procesos de evaluación que permiten optimizar el rendimiento académico, establecer el proceso para el registro estudiantil, con el fin de sistematizar la información académica del alumnado; es decir, revisar, transcribir, organizar, controlar y emitir los documentos probatorios de estudios, tales como certificaciones de calificaciones, constancias de estudios, récords académicos, 
historial académico, cartas de buena conducta, títulos; entre otros, que son requeridos por los alumnos, para asegurar su legalidad y validez ante los organismos competentes.

Igualmente, estas instituciones de estudios requieren velar por la custodia de los expedientes estudiantiles, así como garantizar la veracidad de la información contenida, tramitar las solicitudes de grado de los estudiantes, certificación de títulos, equivalencia de estudios y de reingreso, de acuerdo con los requisitos de admisión y equivalencias establecidas por los reglamentos, resoluciones, acuerdos y demás normas vigentes.

Actualmente las instituciones de educación superior públicas en la Costa Oriental del Lago cuentan con apoyo tecnológico para procesar la información de cada una de las actividades académicas generadas (admisión, evaluación y control de estudios) como horarios, matrícula, nómina de alumnos, modificaciones, notas y actas, bajo un ambiente virtual, es decir vía web.

Sin embargo, se observa con preocupación en el caso de estas instituciones: Universidad del Zulia (LUZ), Instituto Universitario de Tecnología de Cabimas (IUTC) y Universidad Nacional Experimental "Rafael María Baralt" (UNERMB), que los departamentos de control de estudio desarrollan sus actividades con carencia de materiales, falta de mantenimiento en equipos y máquinas.

Según el personal entrevistado que labora en estos departamentos, existe un sistema (software) que no está a la altura de la tecnología actual para el proceso de inscripciones, carga de notas, expedientes, organizar, procesar, clasificar, archivar los datos y documentos relacionados con el historial académico. En algunos casos, hay retrasos debido a la falta de sistema (internet), carecen de plantas eléctricas que garanticen el funcionamiento seguro de los procesos del departamento, cuando el servicio de energía en la región es suspendido, además, falta de formación, capacitación y conocimiento de la malla curricular de cada uno de los programas de estudios por parte del personal que labora en estas áreas, lo cual conlleva a que el alumno sea inscrito indebidamente y que no se mantengan actualizados los datos en los sistemas de control de estudios.

Además, en el proceso de implementación no se cuenta con un stock de materiales de papelería, que son motivo principal para que las actividades que se desarrollan diariamente se vean afectadas por falta de dichos insumos, así como también la falta de mantenimiento que requieren las computadoras, impresoras, fotocopiadoras, entre otros, que allí se usan.

Cabe destacar que, hay falta de orientación e información a la comunidad estudiantil sobre los procesos y normas de los departamentos de control de estudios, lo cual se evidencia en situaciones donde los alumnos no aparecen en la nómina del docente que dicta la unidad curricular, sino que se encuentran inscritos en otras secciones.

Partiendo de las situaciones antes descritas, se puede inferir la existencia de debilidades en las coordinaciones de estos departamentos, que exponen a las instituciones de educación superior públicas en la Costa Oriental del Lago a que su población estudiantil se sienta insatisfecha con los resultados obtenidos a la hora de realizar sus trámites.

En virtud de esta situación surge la siguiente interrogante de investigación: ¿Cómo es la gestión logística en los departamentos de control de estudios de las instituciones públicas 
de educación superior en la Costa Oriental del Lago?

Por tal motivo, el presente estudio tiene como objetivo principal describir la gestión logística en los departamentos de control de estudios de las instituciones públicas de educación superior en la Costa Oriental del Lago.

A lo largo de la historia, la gestión logística ha sido parte fundamental de los procesos administrativos y organizacionales, debido a que es considerada como un conjunto de métodos necesarios para la correcta planificación y gestión de actividades, lo que la convierte en un aliado imprescindible del sector empresarial.

\section{Gestión logística}

Según Carrillo (2013), en una organización la gestión logística es el proceso de planificar, llevar a la práctica y controlar el movimiento y almacenamiento, de forma eficaz y a costos efectivos, de materias primas, productos en fabricación y productos terminados y de la información con ellas relacionada, desde el punto de origen hasta el lugar de consumo, con el fin de actuar conforme a las necesidades del cliente.

De igual forma, Reyes (2007), señala que la gestión logística está enfocada en planificar y coordinar todas las actividades necesarias para conseguir los niveles deseados de servicio y calidad al menor coste posible. También expresa que la logística debe contemplarse como el enlace que une las necesidades de mercado y la actividad operativa de la empresa industrial y esta abarca toda la organización, desde la gestión de materias primas hasta la entrega del producto terminado.

Aunado a lo anterior, Olaya (2007), afirma que en general, la gestión logística se refiere a todos aquellos procesos que facilitan el flujo de bienes y servicios desde su origen al de consumo, teniendo en cuenta a los proveedores de materias y de insumos, fabricantes y a la cadena de distribución, con el fin de satisfacer los requerimientos del consumidor final. Para este autor el propósito de la gestión logística es incrementar los niveles de servicio a los clientes y minimizar los costos.

En este mismo orden de ideas, Hill y Jones (2009), considera la gestión como el proceso de ordenar y emplear conjunto de recursos orientados hacia el logro de metas, para llevar a cabo las tareas de un escenario organizacional. Por su parte, para Council of Supply Chain of Management Professionals, CSCMP (2013), la logística, es aquella parte de la gestión de la cadena de suministro que planifica, implementa y controla el flujo y el almacenamiento eficaz y eficiente de los bienes, servicios e información relacionada desde el punto de origen al de consumo con el objetivo de satisfacer los requerimientos de los consumidores.

Así, la gestión logística puede entenderse como el proceso que permite planificar, organizar, dirigir y controlar todas las actividades de la empresa, que involucra la distribución eficiente de los servicios y productos, desde que llega la materia prima hasta que se distribuye el producto al consumidor, orienta a las organizaciones al logro de las metas y garantiza de esta forma satisfacer a los clientes o consumidores. En la presente investigación la variable gestión logística se medirá a través de las siguientes dimensiones: planificación, organización, ejecución y control. 


\section{Planificación}

La planificación figura como la principal función de la gestión logística por ser la base de las demás. Según Chiavenato (2006), la planificación es la función administrativa que determina de forma anticipada cuáles son los objetivos que deben alcanzarse y qué debe hacerse para cumplirlos. Este autor revela, que se debe empezar por determinar los objetivos y detallar los planes necesarios para alcanzarlos de la mejor manera posible.

Así mismo, Francés (2006, p. 23) señala que la planificación "es un proceso en el cual se definen de manera sistemática los lineamientos estratégicos de la empresa u organización, y se los desarrolla en guías detalladas para la acción, se asignan recursos y se plasman en documentos llamados planes". Por su parte, Ballou (2004), señala que la planificación o planeación logística busca responder las preguntas qué, cuándo y cómo. Además, tiene lugar en tres niveles: estratégica, táctica y operativa. Donde la principal diferencia entre ellas es el horizonte de tiempo para su planificación.

De igual forma, Camisón y Dalmau (2009), indican que la planificación consiste en decisiones anticipadas que van encaminadas a establecer un futuro deseado y la mejor manera de llegar a él. Es decir, ésta permite elegir y fijar las misiones y objetivos de la organización, determinar las políticas, proyectos, programas, procedimientos, métodos, presupuestos, normas como estrategias necesarias para lograrlos, incluyendo, además, la toma de decisiones al tener que escoger entre diversos cursos de acción futuros.

Robbins y Coulter (2010) establecen como elementos de la planificación los siguientes: misión, visión, objetivos y metas, políticas y planes.

\section{Organización administrativa}

Otro elemento a considerar en la gestión logística lo constituye la organización, en esta se decide quién o quiénes llevan a cabo que parte del plan y con qué recursos cuentan. De acuerdo con Chiavenato (2006, p. 148), "la organización significa el acto de organizar, estructurar e integrar los recursos y los órganos involucrados en la ejecución, y establecer las relaciones entre ellos y las atribuciones de cada uno". En este proceso se asignan tareas, recursos, responsabilidades y se asegura que todas las acciones se puedan llevar a cabo al estar debidamente asignadas.

De igual forma, Ballou (2004), plantea que la organización administrativa es la estructura que facilita la creación, la puesta en práctica y la evaluación de los planes. Es el mecanismo formal o informal para distribuir los recursos humanos de tal manera que una empresa alcance sus metas.

En razón de las definiciones anteriores, puede inferirse que la organización implica un grupo de personas, medios, acciones y recursos que se involucran entre sí con todos los procesos de la empresa, de forma organizada, para lograr un determinado propósito.

En la presente investigación la dimensión organización fue medida a través de los siguientes indicadores: estructura organizacional, autoridad, responsabilidad y motivación. 


\section{Ejecución}

Charan y Bossidy (2002), definen la ejecución como el proceso sistemático de discutir los cómo y los qué, cuestionar, dar un seguimiento con tenacidad, asegurar que se rindan cuentas, hacer suposiciones sobre el ámbito de los negocios, evaluar la capacidad organizativa, enlazar la estrategia a las operaciones y a las personas que van a implementar la estrategia, sincronizándolas a sus diversas disciplinas y vincular las recompensas a los resultados.

Por su parte, Chiavenato (2000), indica que la ejecución consiste en poner en marcha lo planificado, está relacionada con la acción y tiene mucho que ver con las personas. Así, la ejecución pone en acción las pautas trazadas durante los procesos de planificación y organización, como también la toma de decisiones.

De igual forma Lépiz (2003), manifiesta que:

La ejecución se refiere a la puesta en práctica de lo planificado, dentro de plazos establecidos y en función de los objetivos propuestos. El éxito de esta acción, depende del control que se llegue a establecer para determinar si los resultados de la ejecución concuerdan con los objetivos propuestos (p. 89).

En este orden de ideas, la ejecución no es más que hacer realidad todo aquello que ha sido planeado, preparado y organizado, a través de la realización de un conjunto de tareas y operaciones que se encuentran establecidas en los planes de trabajo, con el fin de lograr los objetivos planteados. Obviamente la garantía de la ejecución de lo plasmado en los planes de trabajo radica en un adecuado control.

La dimensión ejecución fue medida a través de los siguientes indicadores: sistemas de información, manejo de materiales, inventarios, almacenamiento, transporte y compras.

\section{Control}

Según Robbins y Coulter (2010), el control es el proceso de monitorear las actividades para asegurar que se cumplan como se planifica, corrigiendo cualquier desviación significativa. Además, indican que el control es importante porque ayuda a los gerentes a saber si se están cumpliendo los objetivos organizacionales, y de no ser así, las razones por las que no se están cumpliendo.

Igualmente, uno de los beneficios que genera el control consiste en permitir la inspección de todas las actividades que se están realizando dentro de la organización, con el propósito de conocer si se ejecutan de forma adecuada y de no ser así, corregir cualquier error a tiempo.

De acuerdo con Boland y col. (2007), la función del control permite que, en toda organización, se pueda comprobar en forma permanente en qué medida sus planes se están cumpliendo y asegurarse que existan altas probabilidades de alcanzar los objetivos que se ha fijado. Asimismo, Mora (2010, p. 75) indica que "el control tiene por objeto cerciorarse de que los hechos vayan de acuerdo con los planes establecidos". Señala que el control 
tiene los siguientes factores: relación con lo planeado, medición, detectar desviaciones y establecer medidas correctivas.

De igual manera, Camisón y Dalmau (2009) aseveran que, a través del proceso de control, los gerentes establecen y diseñan sistemas de medida y se aseguran de que la organización alcance los objetivos planificados. Este permite hacer una comparación entre los resultados que se van obteniendo con los objetivos planeados y, en la medida en que existan desviaciones importantes, se debe analizar las causas de dichas desviaciones para idear e implantar las medidas correctivas para reconducir al sistema hacia sus objetivos

Por su parte, Ballou (2004) afirma que el control es el proceso que permite regular el desempeño planeado en correspondencia con los objetivos trazados por la organización, tal como se especifica en la Figura 1.

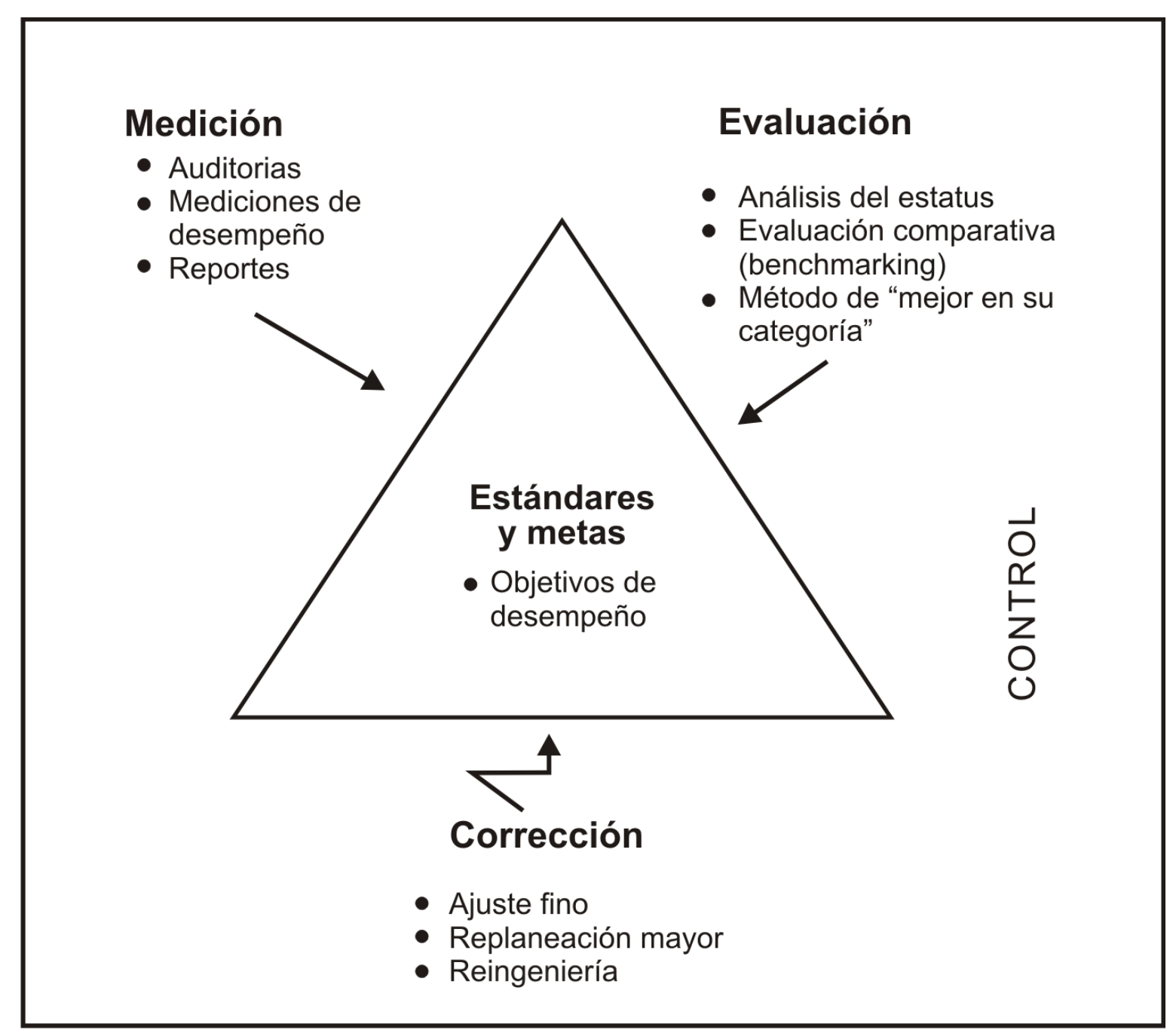

Figura 1. Triangulo del control. (Fuente: Ballou, 2004). 
De igual manera, los autores antes citados coinciden en que el proceso de control es el seguimiento que se le hace a las actividades dentro de las organizaciones para mantener regulado los objetivos planteados en el proceso de planificación, lo cual permite detectar y corregir a tiempo, las desviaciones que se presentan en las organizaciones o empresas.

A objeto de analizar la dimensión control se tomaron como indicadores: auditorias, desempeño y servicio al cliente.

\section{MÉTODO}

L

a investigación se consideró de tipo descriptiva bajo el diseño de campo, transeccional, la población estuvo conformada por doce (12) trabajadores adscritos a los departamentos de control de estudios de las instituciones públicas de educación superior en la Costa Oriental del Lago: Universidad del Zulia (LUZ) Núcleo COL, Instituto Universitario de Tecnología de Cabimas (IUTC) y la Universidad Nacional Experimental "Rafael María Baralt"(UNERMB), La técnica de recolección de datos utilizada fue la encuesta. El instrumento lo constituyó un cuestionario conformado por 56 ítems con alternativas de respuestas en escalas tipo Likert. Para determinar el grado de confiabilidad del instrumento se aplicó el Coeficiente de Alfa Cronbach, obteniéndose un índice de confiabilidad de 0,973 de muy alta confiabilidad. Para el análisis de la información obtenida, se emplearon como técnicas de tratamiento de datos la estadística descriptiva, específicamente la medida de tendencia central media, cuyo baremo de medición para la interpretación de la media se especifica en la Tabla 1.

Tabla 1. Baremo de medición para interpretación de la media.

\begin{tabular}{|c|c|c|c|c|}
\hline Valor & Alternativa & Intervalo & Categoría & Descripción \\
\hline 5 & Muy de acuerdo (MDA) & $4,21-5,00$ & Muy alto & $\begin{array}{l}\text { Muy alto cumplimiento de la variable, } \\
\text { dimensión o indicador. }\end{array}$ \\
\hline 4 & De acuerdo (DA) & $3,41-4,20$ & Alto & $\begin{array}{l}\text { Alto cumplimiento de la variable, dimensión } \\
\text { o indicador, presentando posibles mejoras. }\end{array}$ \\
\hline 3 & $\begin{array}{l}\text { Ni de acuerdo ni en } \\
\text { desacuerdo (NDANED) }\end{array}$ & $2,61-3,40$ & Moderado & $\begin{array}{l}\text { Cumplimiento de la variable, dimensión o } \\
\text { indicador con ciertas debilidades. }\end{array}$ \\
\hline 2 & En desacuerdo (ED) & $1,81-2,60$ & Bajo & $\begin{array}{l}\text { Bajo cumplimiento de la variable, dimensión } \\
\text { o indicador presentando altas debilidades. }\end{array}$ \\
\hline 1 & $\begin{array}{l}\text { Muy en desacuerdo } \\
\text { (MED) }\end{array}$ & $1,00-1,80$ & Muy bajo & $\begin{array}{l}\text { Muy bajo cumplimiento de la variable, } \\
\text { dimensión o indicador podría decirse que es } \\
\text { poco o nada. }\end{array}$ \\
\hline
\end{tabular}




\section{RESULTADOS}

$\mathrm{L}$ a variable gestión logística en los departamentos de control de estudios de las instituciones públicas de educación superior en la Costa Oriental del Lago, fue analizada a través de las dimensiones: planificación, organización, ejecución y control.

Con relación a la dimensión planificación, evaluada bajo los indicadores: misión, visión, objetivos - metas, políticas y planes, se obtuvo una media de 2,55, que según el baremo de interpretación (tabla 1) le ubica en la categoría bajo cumplimiento, tal como puede observarse en la tabla 2. Este resultado evidencia que existe un bajo cumplimiento en la dimensión planificación. Igual ubicación alcanzaron los indicadores: visión $(2,45)$, objetivos - metas $(2,50)$ y planes $(2,59)$, mientras que el indicador políticas $(3,40)$ se ubicó en una categoría de moderado, en tanto que el indicador misión $(1,80)$ se posicionó en una categoría de un muy bajo cumplimiento.

Tabla 2. Media de la dimensión planificación.

\begin{tabular}{lcl}
\hline \multicolumn{1}{c}{ Indicadores } & Media & \multicolumn{1}{c}{ Categoría } \\
\hline Misión & 1,80 & Muy bajo cumplimiento \\
Visión & 2,45 & Bajo cumplimiento \\
Objetivos - metas & 2,50 & Bajo cumplimiento \\
Políticas & 3,40 & Moderado cumplimiento \\
Planes & 2,59 & Bajo cumplimiento \\
\hline Resumen de la dimensión & $\mathbf{2 , 5 5}$ & Bajo cumplimiento \\
\hline
\end{tabular}

En tal sentido, los procesos de planificación de los departamentos de control de estudios de las instituciones públicas de educación superior objeto de este estudio se desarrollan de manera deficiente en sus componentes de políticas, visión, objetivos- metas y planes y muy deficiente con relación a la misión, situación que dificulta la gobernabilidad y la direccionalidad de las mismas y desagregan el esfuerzo colectivo en la búsqueda de la dirección trazada.

Teniendo en consideración que la visión y misión de una organización constituyen el punto de partida para el desarrollo de estrategias que apunten al crecimiento organizacional, llama la atención el comportamiento de los indicadores que conforman la dimensión planificación al alcanzar resultados en las categorías desde muy bajos a bajos, lo cual pone de manifiesto improvisaciones en el proceso de planificación que desarrollan las instituciones en estudio.

En efecto, no es de extrañar las situaciones que presentan estas instituciones en sus procesos, ya que una visión y misión de bajo cumplimiento impacta las políticas que definen 
cuáles son los principios de estas organizaciones y cuáles son los modos de trabajo y estándares de actuación en cada una de las situaciones que se presentan en el día a día, así como el establecimiento de objetivos y planes a corto, mediano y largo plazo.

Estos resultados son opuestos con lo expresado por Camisón y Dalmau (2009), quienes indican que la planificación encamina a la organización a establecerse en un futuro deseado, por lo tanto, permite fijar la misión y objetivos, determinar las políticas y planes que permitan cumplir con los objetivos planteados.

De igual manera, autores como Chiavenato (2006), Francés (2006), entre otros, coinciden con lo antes planteado al considerar la función planificación como la base o principal fundamento de la gestión logística en toda organización, al permitirle decidir con anticipación lo que se quiere lograr en el futuro y el cómo se va a lograr con la finalidad de satisfacer los requerimientos de cliente.

Con respecto a la dimensión organización de la gestión logística en los departamentos de control de estudios de las instituciones públicas de educación superior en la Costa Oriental del Lago, la Tabla 3, muestra los resultados que arrojó el análisis de dicha dimensión a través de los indicadores estructura organizacional, autoridad, responsabilidad y motivación.

Tabla 3. Media de la dimensión organización.

\begin{tabular}{lcl}
\hline \multicolumn{1}{c}{ Indicadores } & Media & \multicolumn{1}{c}{ Categoría } \\
\hline Estructura organizacional & 3,40 & Moderado cumplimiento \\
Autoridad & 2,85 & Moderado cumplimiento \\
Responsabilidad & 2,81 & Moderado cumplimiento \\
Motivación & 2,00 & Bajo cumplimiento \\
\hline Resumen de la dimensión & $\mathbf{2 , 7 6}$ & Moderado cumplimiento \\
\hline
\end{tabular}

Como puede apreciarse en la Tabla 3, la dimensión organización presenta una media de 2,76, que según el baremo de interpretación de la media la ubica en una categoría de moderado cumplimiento, por lo que puede deducirse que en los departamentos de control de estudios de las instituciones estudiadas existe un moderado nivel de organización. Muy a pesar que los diferentes aspectos que componen esta dimensión tienen como resultado moderado cumplimiento organizacional que permite la asignación de tareas, definir las líneas de autoridad y sus responsabilidades; llama la atención que la motivación resultara de bajo cumplimiento, no obstante, da explicación al resultado total de la dimensión, al ser la motivación considerada como fuerza vital que moviliza procesos e individualidades en el logro de las metas.

Asimismo, estos resultados son opuestos con lo planteado por Boland y col. (2007, p. 60), cuando expone que la estructura organizacional "es la forma en que se distribuyen las personas y las tareas en una organización a fin de hacer real el logro de sus objetivos". 
En tal sentido, se puede decir que la estructura organizacional es de suma importancia ya que representa la estructura en la cual se visualizan las operaciones, quiénes son los responsables de llevar a cabo las actividades claves de las empresas y cuánta autoridad sustentan sus cargos.

De igual manera, Chiavenato (2006) sostiene que en toda organización deben estar claramente definidas las líneas de autoridad, tareas y funciones, lo cual garantiza el éxito en las acciones emprendidas.

En lo que corresponde a la dimensión ejecución de la gestión logística, cuyos indicadores fueron: sistemas de información, manejo de materiales, inventarios, almacenamiento, transporte y compras, manifestados en la Tabla 4, en estas instituciones la dimensión ejecución presentó una media de 2,51 que al compararla con el baremo de interpretación de la media dio como resultado bajo cumplimiento. Iguales comportamientos presentaron los indicadores almacenamiento $(2,00)$, transporte $(2,40)$ y compras $(2,55)$; sistema de información $(2,85)$, se ubicó en una posición de moderado cumplimiento; mientras que manejo de materiales $(3,45)$ e inventarios $(1,80)$ ubicaron posiciones contrapuestas de alto cumplimiento y muy bajo cumplimiento respectivamente.

Tabla 4. Media de la dimensión ejecución.

\begin{tabular}{lcl}
\hline \multicolumn{1}{c}{ Indicadores } & Media & \multicolumn{1}{c}{ Categoría } \\
\hline Sistemas de información & 2,85 & Moderado cumplimiento \\
Manejo de materiales & 3,45 & Alto cumplimiento \\
Inventarios & 1,80 & Muy bajo cumplimiento \\
Almacenamiento & 2,00 & Bajo cumplimiento \\
Transporte & 2,40 & Bajo cumplimiento \\
Compras & 2,55 & Bajo cumplimiento \\
\hline Resumen de la dimensión & $\mathbf{2 , 5 1}$ & Bajo cumplimiento \\
\hline
\end{tabular}

Aun cuando la ejecución es considerada un proceso dinámico donde se convierte en realidad lo planeado, preparado y organizado y sus miembros contribuyen a alcanzar los objetivos, la información proporcionada por los resultados de esta dimensión mostraron que las actividades en las instituciones en estudio se llevan a cabo en bajo cumplimiento, lo cual guarda coherencia con el nivel de cumplimiento alcanzado por la dimensión planificación de la gestión logística que se lleva a cabo en estas organizaciones.

Estos resultados se contraponen con lo expuesto por Charan y Bossidy (2002), quienes consideran que en todo proceso de ejecución es de suma importancia llevar a cabo un proceso tenaz de seguimiento, asegurar que se rindan cuentas, evaluar la capacidad organizativa, enlazar la estrategia a las operaciones y a las personas que van a implementar la estrategia, sincronizándolas a sus diversas disciplinas y vincular las recompensas a los resultados. 
De igual manera, Lépiz (2003), afirma que para que las organizaciones lleven a cabo de manera exitosa la función de ejecución, deben establecer controles que les permitan determinar si los resultados de la ejecución concuerdan con los objetivos propuestos.

En relación con el comportamiento de la dimensión control de la gestión logística en los departamentos de control de estudios de las instituciones públicas de educación superior en la Costa Oriental del Lago, cuya media fue de 2,86 que al compararlo con el baremo de interpretación de la media la ubicó en la categoría de moderado cumplimiento que permite inferir que existe un moderado control sobre los procesos inherentes a esta dimensión, como son auditorias $(3,20)$, desempeño $(2,80)$, mientras que el indicador servicio al cliente $(2,60)$ se ubicó en la categoría de bajo cumplimiento. (Tabla 5).

Tabla 5. Media de la de la dimensión control.

\begin{tabular}{lcl}
\hline \multicolumn{1}{c}{ Indicadores } & Media & \multicolumn{1}{c}{ Categoría } \\
\hline Auditorias & 3,20 & Moderado cumplimiento \\
Desempeño & 2,80 & Moderado cumplimiento \\
Servicio al cliente & 2,60 & Bajo cumplimiento \\
\hline Resumen de la dimensión & $\mathbf{2 , 8 6}$ & Moderado cumplimiento \\
\hline
\end{tabular}

Los resultados manifestados en la Tabla 5 están en oposición a lo expuesto por Boland y col (2007), quienes afirman que el control permite comprobar si los planes de la organización se están cumpliendo y de esta forma asegurarse que los objetivos que se han fijado se puedan alcanzar. En tal sentido, a través de los resultados arrojados en esta dimensión, se pudo inferir que, el moderado cumplimiento del control en esta institución generó un impacto negativo en los clientes dando paso a diversos conflictos entre la comunidad que en ellas conviven.

Seguidamente los resultados de la variable gestión logística, presentes en las dimensiones planificación, organización, ejecución y control, expresados en la Tabla 6.

Tabla 6. Media de la variable gestión logística.

\begin{tabular}{lcl}
\hline \multicolumn{1}{c}{ Indicadores } & Media & \multicolumn{1}{c}{ Categoría } \\
\hline Planificación & 2,55 & Bajo cumplimiento \\
Organización & 2,76 & Moderado cumplimiento \\
Ejecución & 2,51 & Bajo cumplimiento \\
Control & 2,86 & Moderado cumplimiento \\
\hline Resumen de la variable & $\mathbf{2 , 6 7}$ & Moderado cumplimiento \\
\hline
\end{tabular}


Tal como muestra la Tabla 6, la media de la variable gestión logística fue de 2,67 que al compararlo con el baremo de interpretación de la media dio como resultado moderado cumplimiento, lo que indica moderado cumplimiento de la gestión logística en los departamentos de control de estudios de las instituciones públicas de educación superior en la Costa Oriental del Lago.

Estos resultados, fueron opuestos a lo planteado por el Consejo de Supply Chain Management Professionals CSCMP (2013), quien afirma que la gestión logística se encarga de planificar, ejecutar y controlar el funcionamiento eficaz y eficiente del almacenamiento de bienes, servicios e información. De igual manera, Reyes (2007), señala que la gestión logística involucra toda la organización y está enfocada en planificar y coordinar todas las actividades necesarias para conseguir los niveles deseados de servicio y calidad al menor coste posible.

En razón de los resultados en este estudio se pudo aseverar que la gestión logística en los departamentos de control de estudios de las instituciones públicas de educación superior en la Costa Oriental del Lago presentó un moderado cumplimiento en los procesos de planificación, organización, ejecución y control de los bienes o servicios.

\section{CONCLUSIONES}

$\longrightarrow$ on relación a la dimensión planificación de la gestión logística en los departamentos de control de estudios de las instituciones públicas de educación superior en la Costa Oriental del Lago, se concluye que las mismas presentan un bajo cumplimiento en su planificación, por lo que se puede inferir que la actividad de sus departamentos se desarrolla de manera deficiente en sus componentes de políticas, visión, objetivos- metas y planes y muy deficiente con relación a la misión.

Teniendo en cuenta que, es a través de la visión y la misión como se delinea el curso a seguir y las estrategias que permiten establecer los objetivos, así como los valores que rigen el comportamiento de las comunidades universitarias en referencia, una alta deficiencia en este aspecto dificulta la gobernabilidad y la direccionalidad de las mismas y desagrega el esfuerzo colectivo en la búsqueda de la dirección trazada, de igual manera, impacta las políticas que definen cuáles son los principios de estas organizaciones y cuáles son los modos de trabajo y estándares de actuación en cada una de las situaciones que se presentan en el día a día.

En cuanto a la dimensión organización, cuyo resultado le ubica en una posición de un moderado nivel de organización, se infiere que entre los miembros de estas instituciones se presentan dificultades para trabajar juntos de manera óptima, alcanzar las metas fijadas durante el proceso de planificación, así como respetar las respectivas líneas de mando, mediano cumplimiento en la asignación de tareas y responsabilidades.

Con relación a la dimensión control que alcanzó un resultado de moderado cumplimiento, se concluye que existe un moderado monitoreo de las actividades logísticas, lo que excede en el bajo cumplimiento de los planes y la poca capacidad de respuesta oportuna cuando se presentan eventos no deseados que conllevan a interrupciones de los procesos, lo cual impacta la efectividad de los procesos logísticos y la calidad del servicio brindado al cliente.

Para finalizar, la variable gestión logística de las instituciones estudiadas se desarrolla con un moderado nivel de cumplimiento. 


\section{REFERENCIAS}

Ballou, R. (2004). Logística. Administración de la cadena de suministro. 5ta edición, México, Editorial Pearson educación.

Boland, L.; Carro, F.; Stancatti, M.; Gismano, Y., y Banchieri, L. (2007). Funciones de la administración. Teoría y Práctica. Argentina, editorial de la Universidad Nacional del Sur

Camisón, C., y Dalmau, J. (2009) Introducción a los negocios y su gestión. Madrid España, Editorial Pearson Educación, S.A.

Casassus, J. (2002). Problemas de la Gestión Educativa en América Latina: la tensión entre los paradigmas de tipo A y el tipo B. Disponible en: http://www. rbep.inep.gov.br/index.php/emaberto/ article/viewFile/2167/2136. [Consulta 20/06/2016 a las 8:34 pm]

Charan, R., y Bossidy, L. (2002). Execution: The discipline of getting things done (Ejecución: La disciplina de lograr que las cosas ocurran). 1ra edición, New York, editorial Crown Publishing Group.

Casanova, A., y Cuatrecasas, L. (2010). Logística empresarial. Sexta edición. Ediciones Gestión 2000 S.A. España

Carrillo (2013): un enfoque para la evaluación, integración y mejora de los procesos logísticos. Sevilla, España, Instituto Andaluz de Tecnología

Chiavenato, I. (2000). Administración de recursos humanos. 5ta edición, Bogotá Colombia, Editorial Mcgraw-Hill Interamericana, S.A.

Chiavenato, I. (2006) Introducción a la teoría general de la administración. 7ma edición, México, Editorial Mcgraw-Hill/ Interamericana Editores, S.A. de C.V.
CSCMP (2013). Council of Supply Chain of Management Professionals, Consejo de profesionales de gestión de la cadena de suministro. En: Supply chain management terms and glossary. Compilado por: Vitaset K. Agosto (2013)

Francés, A. (2006). Estrategias y planes para la empresa, con el cuadro de mando integral. 1ra edición, Pearson educación de México S.A.

Hill, Ch., y Jones, G. (2009). Administración estratégica. 8va edición, México, Editorial Mcgraw-Hill/Interamericana Editores, S.A. de C.V.

Lépiz, C. (2003).La administración y planificación como procesos. 5ta reimpresión de la 1ra edición, Costa Rica, Editorial Universidad Estatal a Distancia EUNED

Mora, L. (2010). Gestión logística integral. Las mejores prácticas en la cadena de abastecimientos. Bogotá. Colombia, Ecoe Ediciones

Olaya, M. (2007). Módulo de logística industrial. lera edición, Universidad Nacional Abierta y a Distancia UNAD, Bogotá Colombia

Rementeria, A. (2008). Concepto de gestión. Editorial Universidad Bolivariana, Santiago de Chile

Reyes, A. (2007). Módulo de logística industrial. Universidad Nacional Abierta y a Distancia UNAD, Bogotá Colombia, editorial Aricorp

Robbins, S. y Coulter, M. (2010). Administración. 10ma edición, México, editorial Pearson 